\title{
Wind noise reduction in a non-porous subsurface windscreen
}

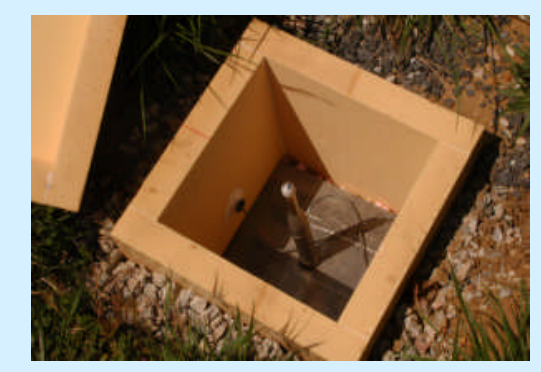

Allan J. Zuckerwar

Analytical Services and Materials, Hampton, VA 23666

and

Qamar A. Shams and H. Keith Knight

NASA Langley Research Center, Hampton, VA 23681

$164^{\text {th }}$ Meeting of the Acoustical Society of America

October 22, 2012 


\section{Profile of horizontal wind}

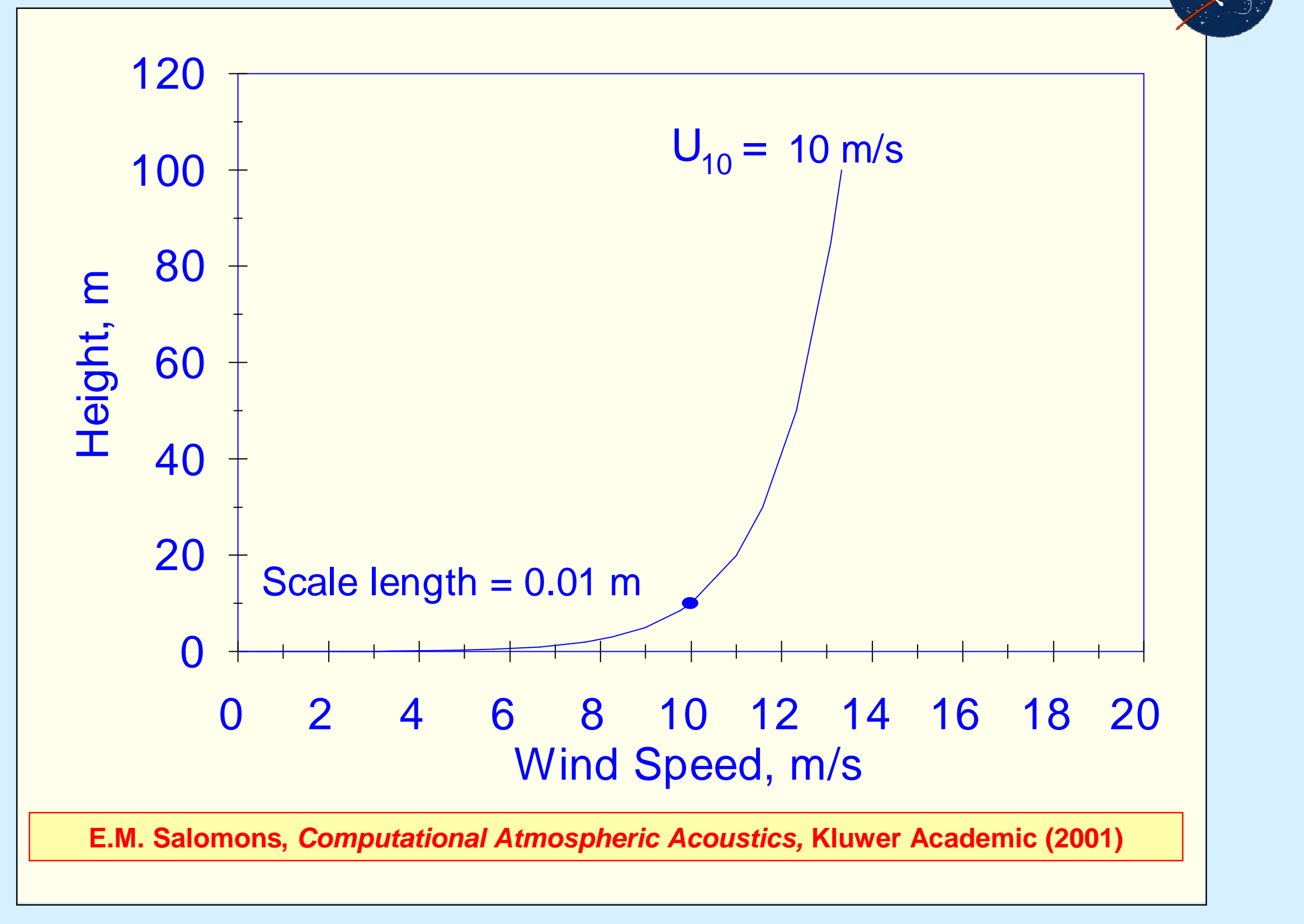




\section{Test configurations}
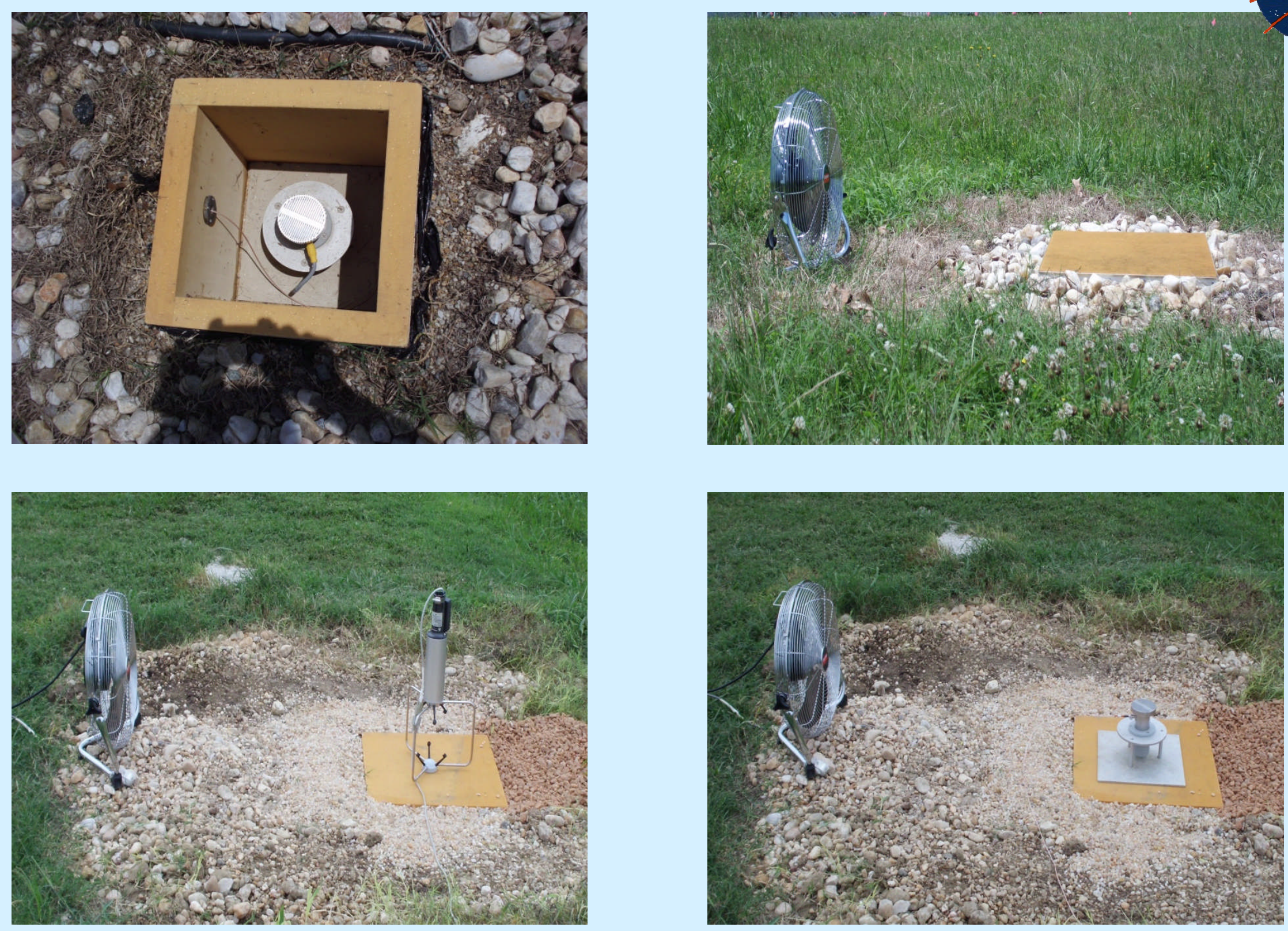


\section{Wind noise levels: $8 \mathrm{lb}$ foam}

Wind speed $3 \mathrm{~m} / \mathrm{s}$

Wind speed $5 \mathrm{~m} / \mathrm{s}$
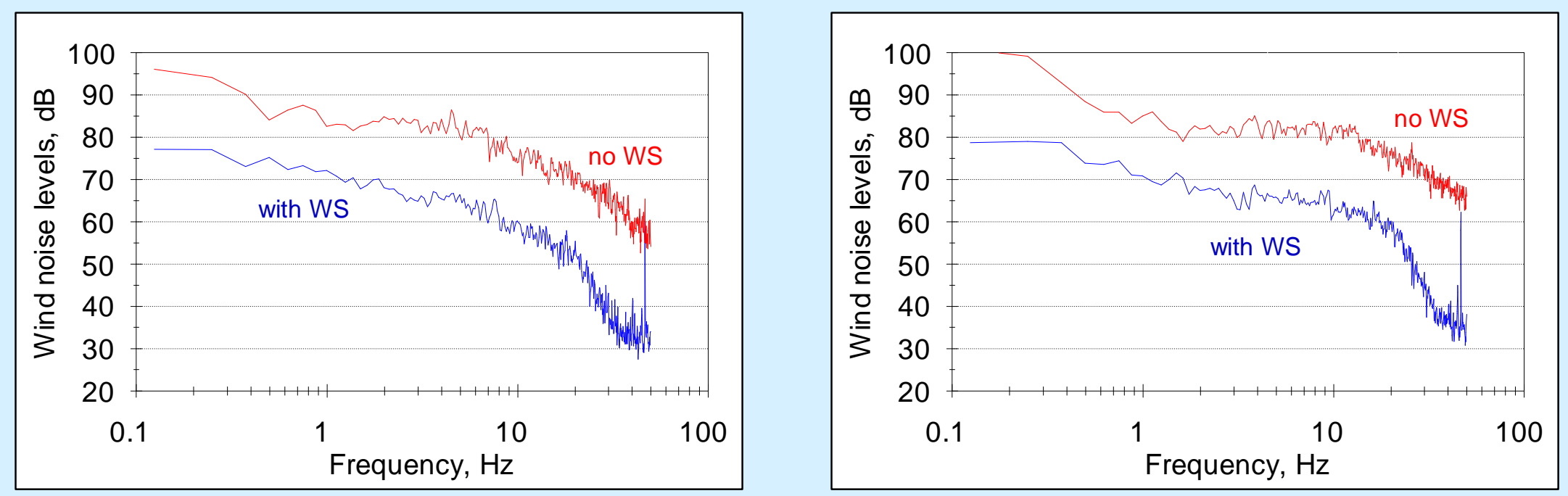

Wind speed $7 \mathrm{~m} / \mathrm{s}$

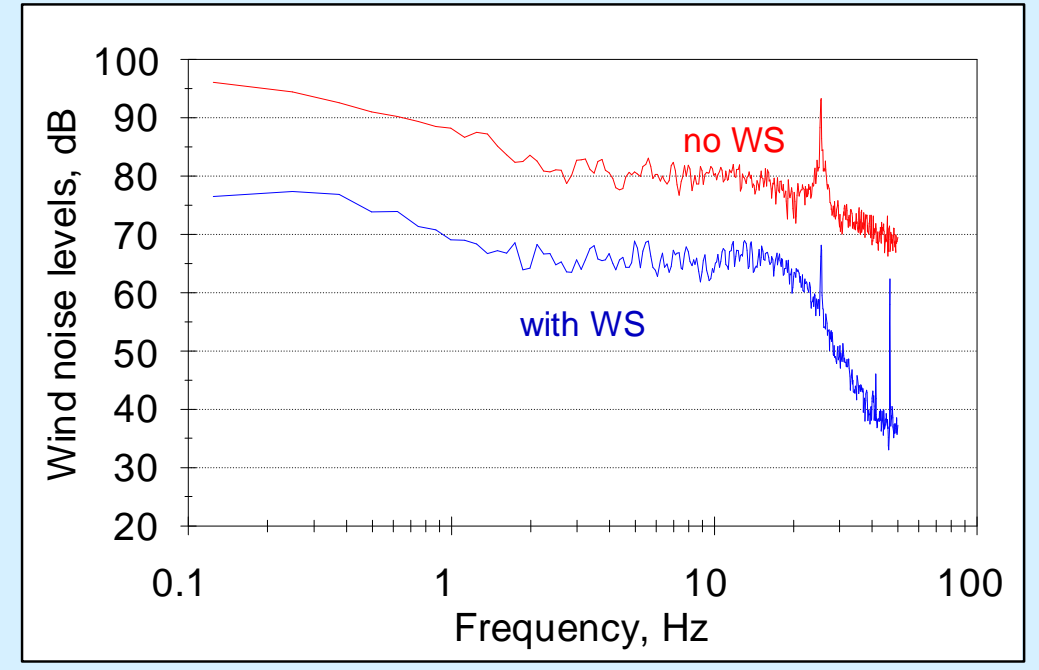




\section{Wind noise reduction: $8 \mathrm{lb}$ foam}

Wind speed $5 \mathrm{~m} / \mathrm{s}$
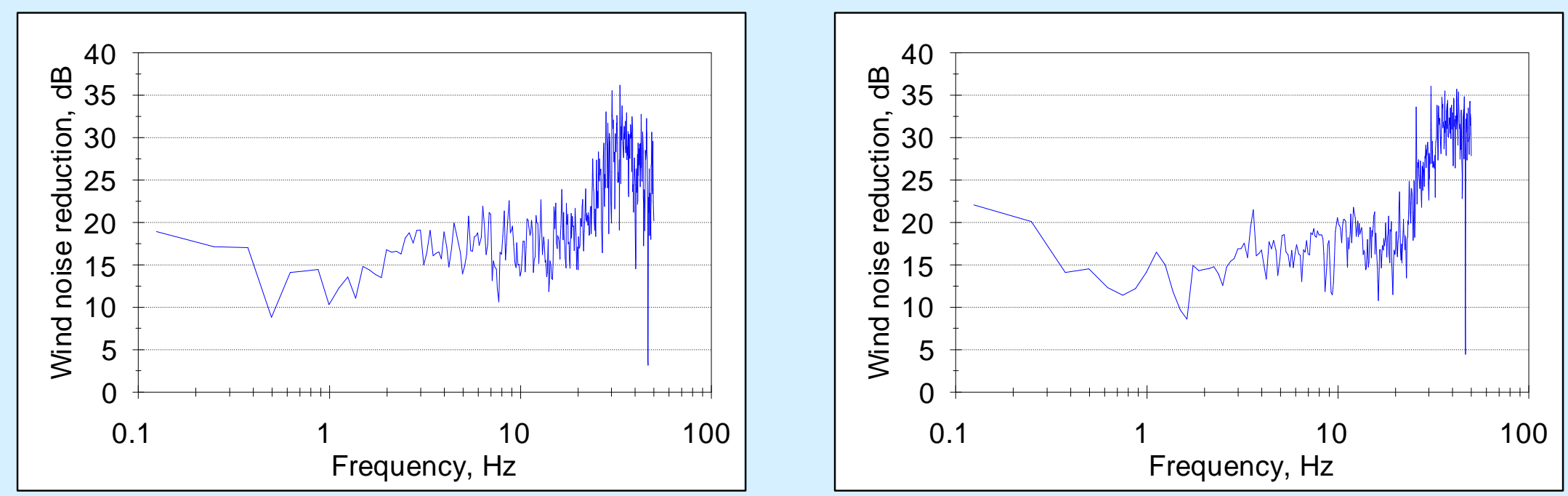

Wind speed $7 \mathrm{~m} / \mathrm{s}$

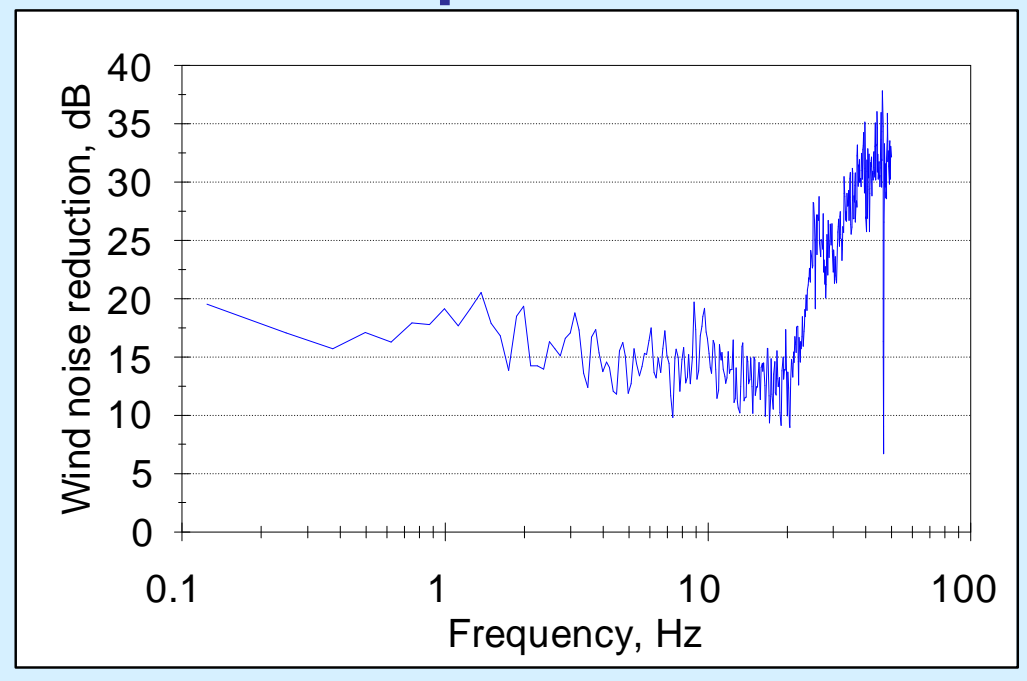




\section{Wind noise reduction: $4 \mathrm{lb}$ foam}

Wind speed $5 \mathrm{~m} / \mathrm{s}$
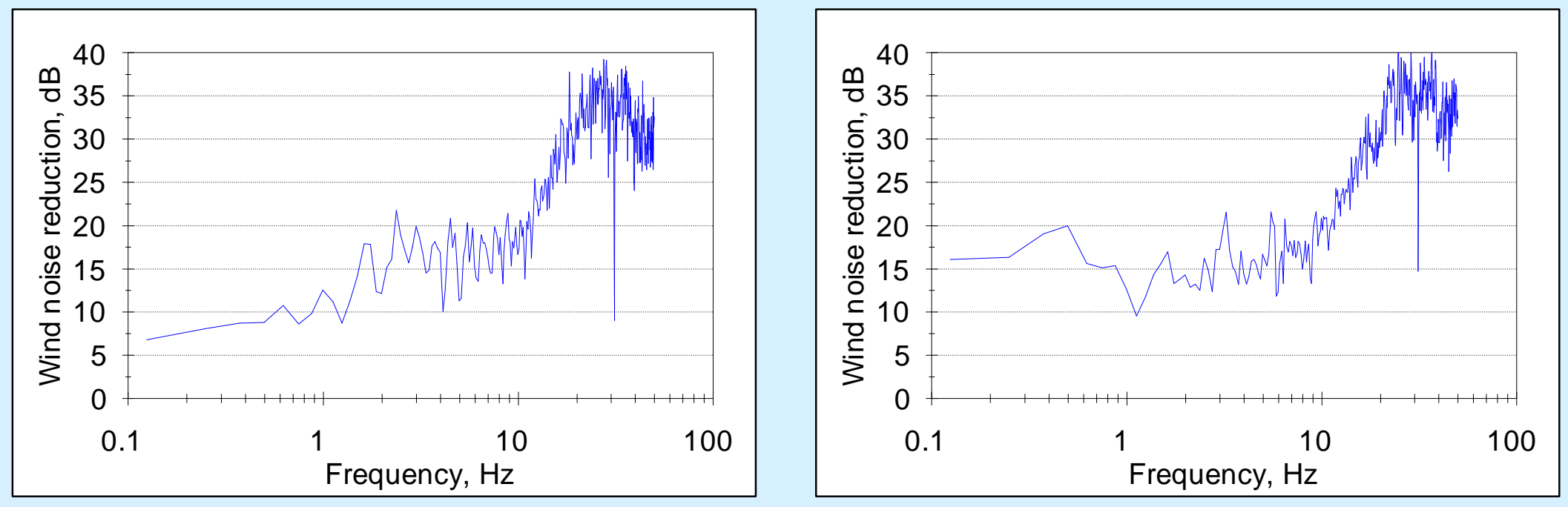

Wind speed $7 \mathrm{~m} / \mathrm{s}$

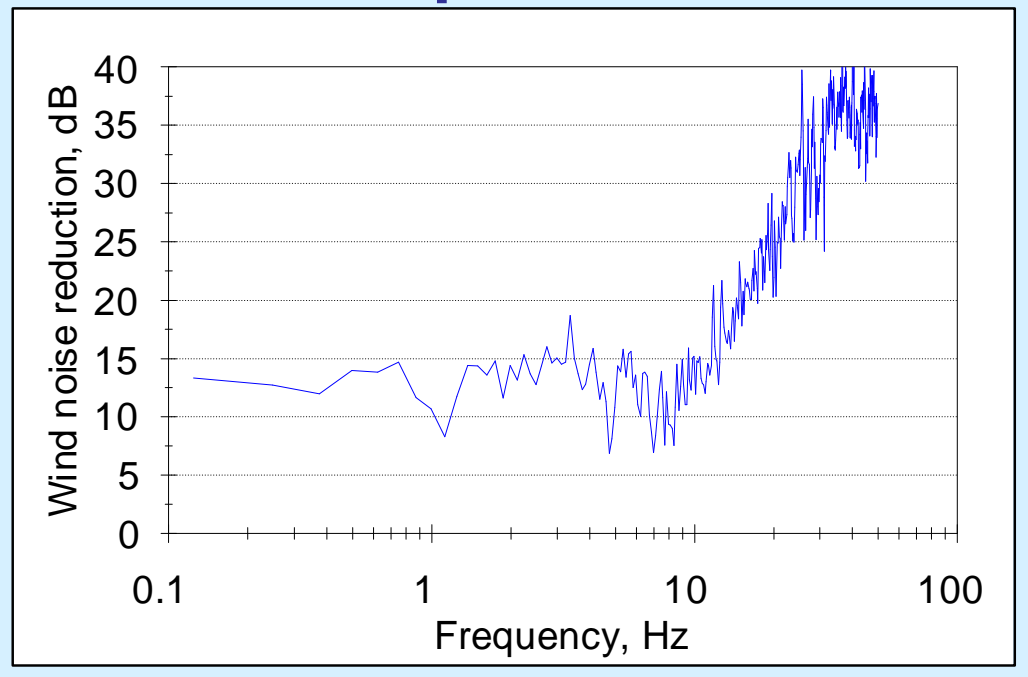




\section{Wind noise reduction: $15 \mathrm{lb}$ foam \\ Wind speed $3 \mathrm{~m} / \mathrm{s}$ \\ Wind speed $5 \mathrm{~m} / \mathrm{s}$}
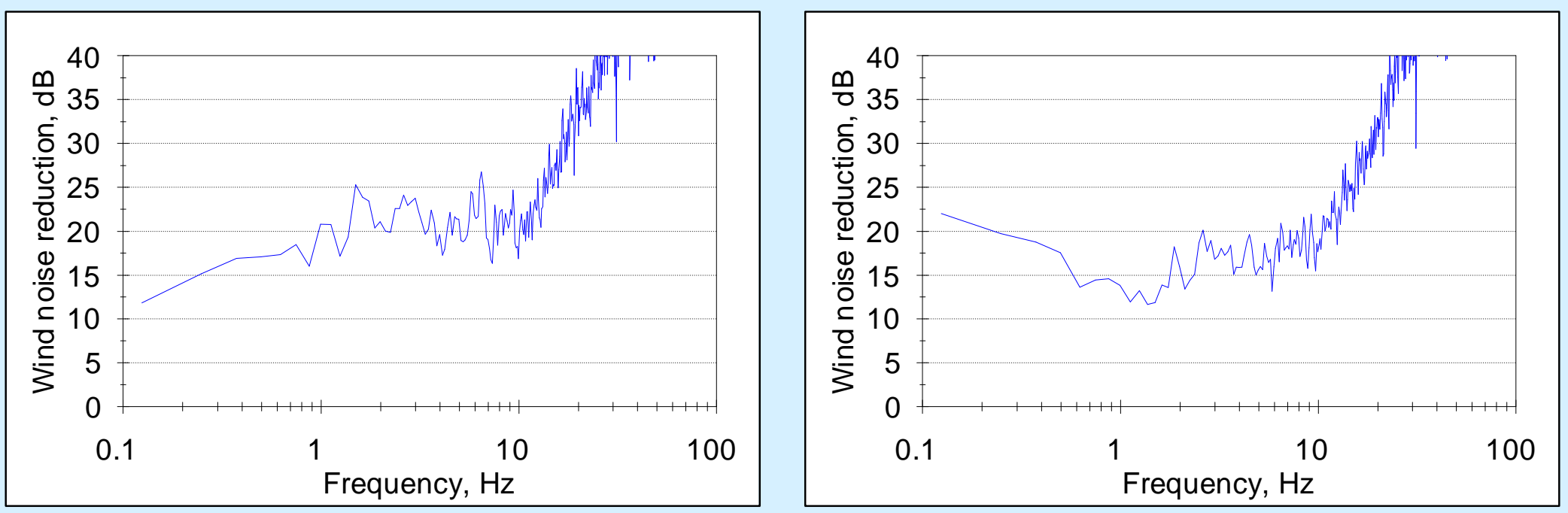

Wind speed $7 \mathrm{~m} / \mathrm{s}$

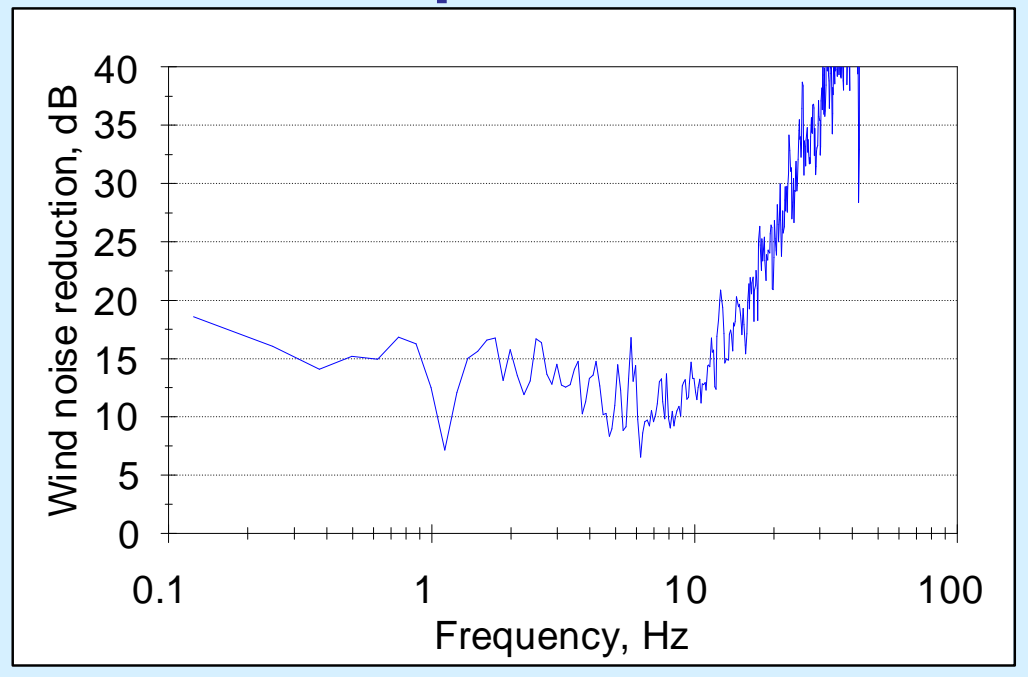




\section{Wind Noise Reduction: Chaparral Physics Helmericks, Marriott, \& Olson 2008}

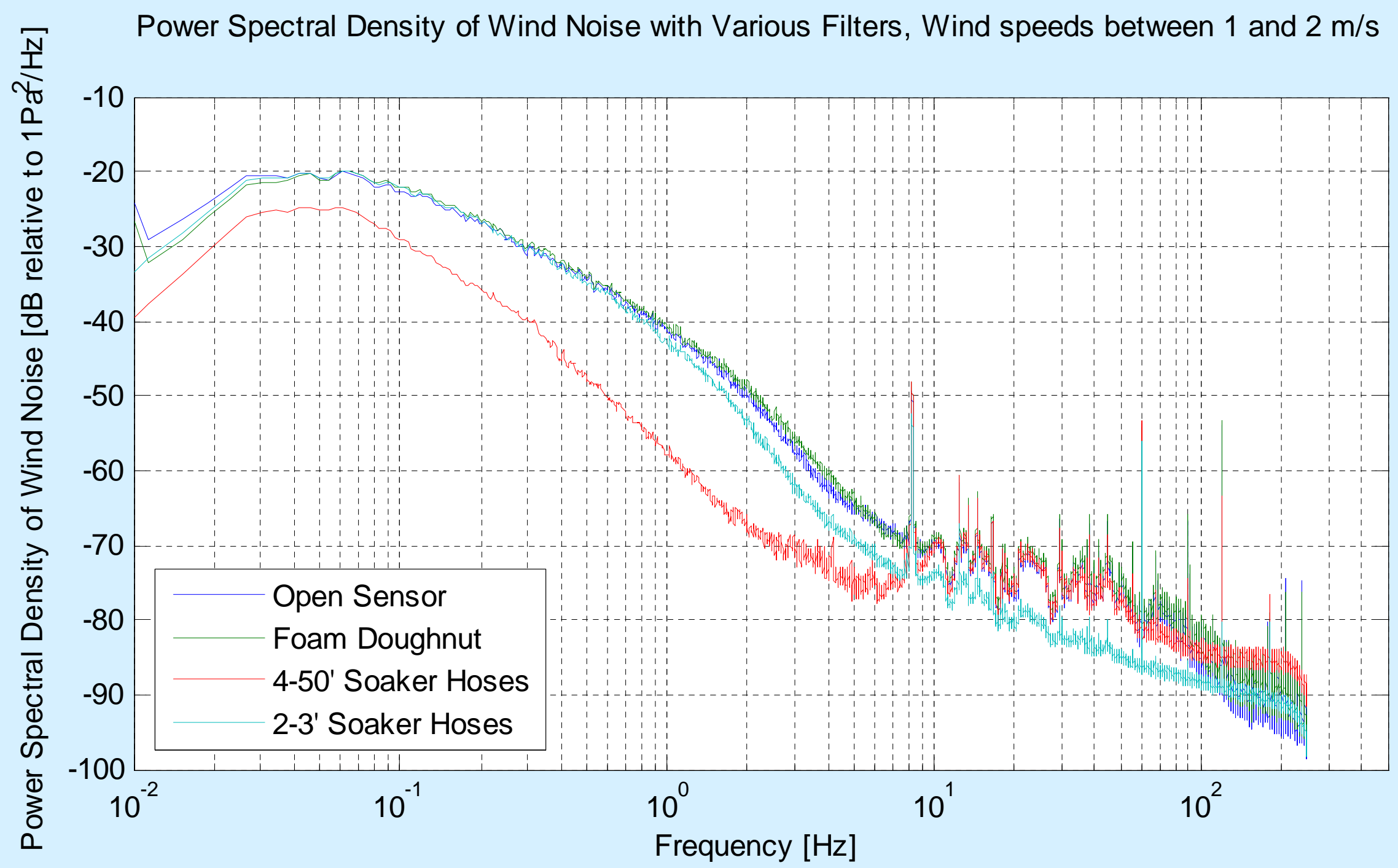




\section{Wind noise reduction: Chaparral Physics $4 \times 50 \mathrm{ft}$ soaker hoses}

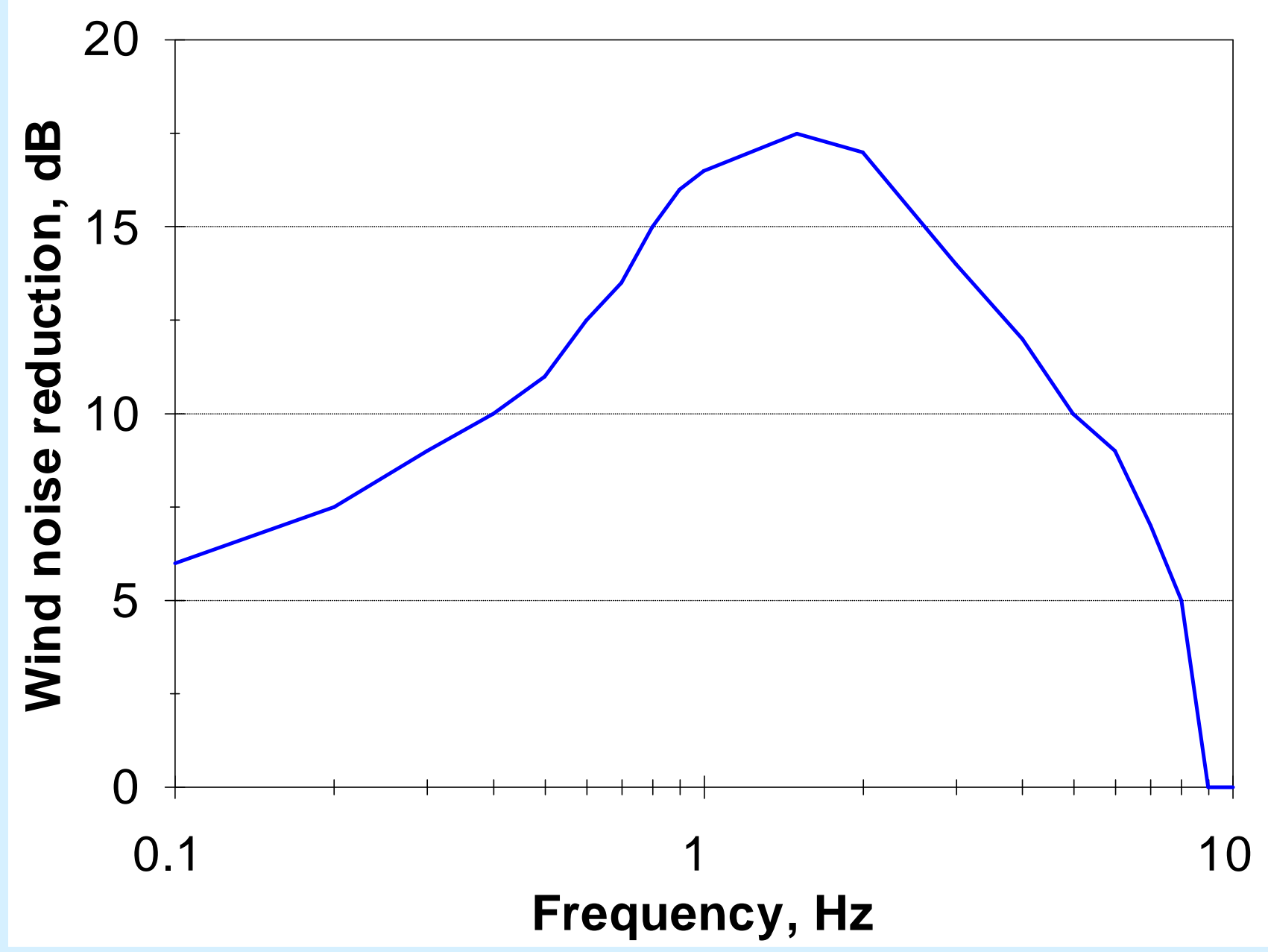




\section{Transmission test: $8 \mathrm{lb}$ foam}

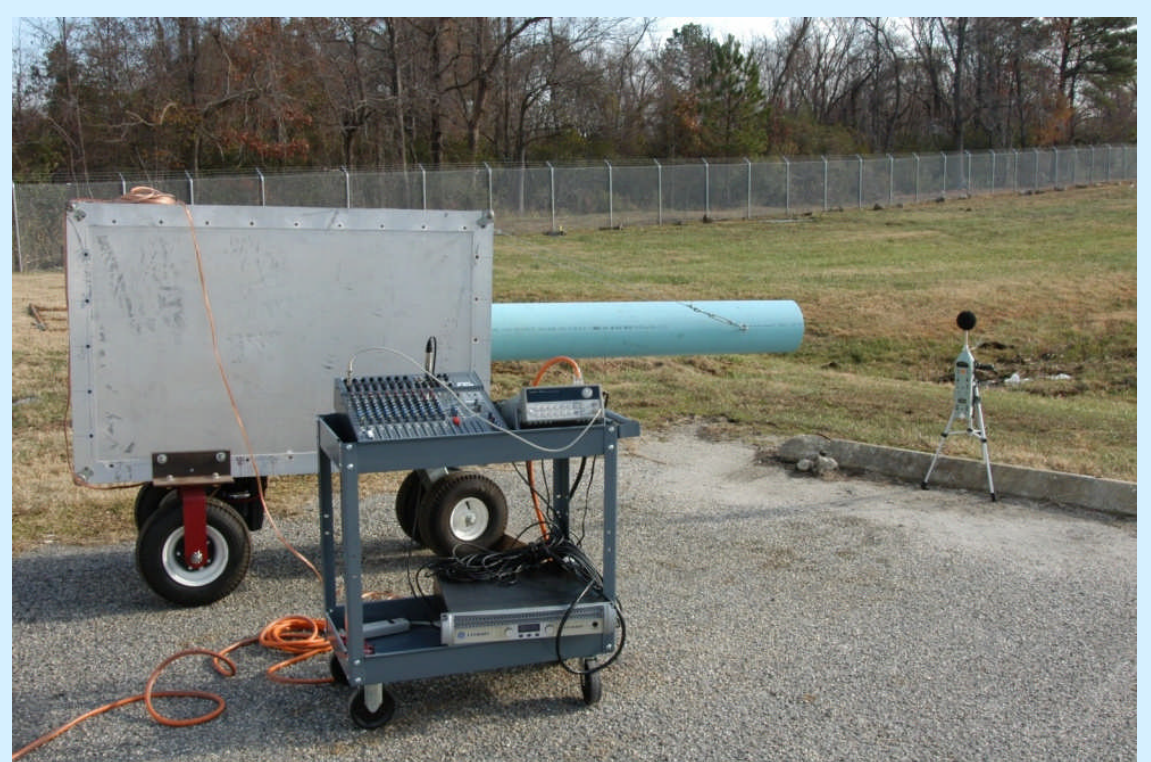

Frequency: $8.55 \mathrm{~Hz}$ SPL @ 1 m: $101 \mathrm{~dB}$

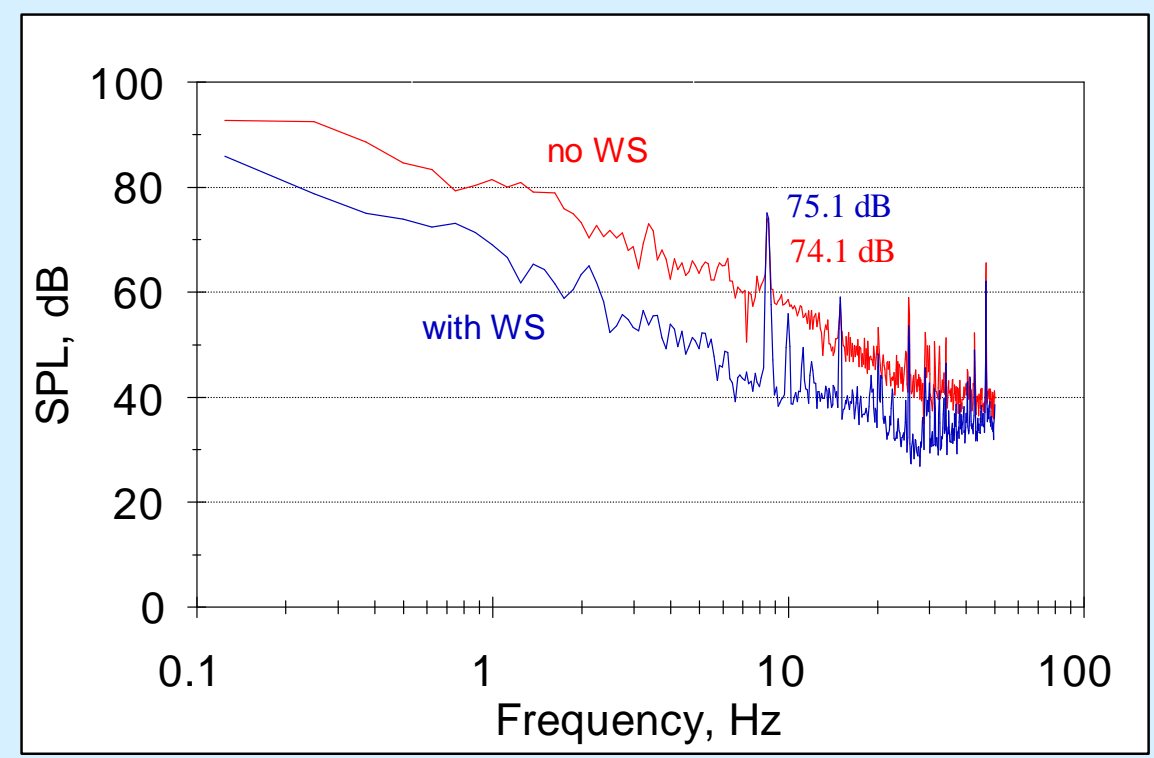

Net gain $1.0 \mathrm{~dB}$

Distance to WS: $21.3 \mathrm{~m}$ (70 ft) 


\section{Transmission test}

$4 \mathrm{lb}$ foam

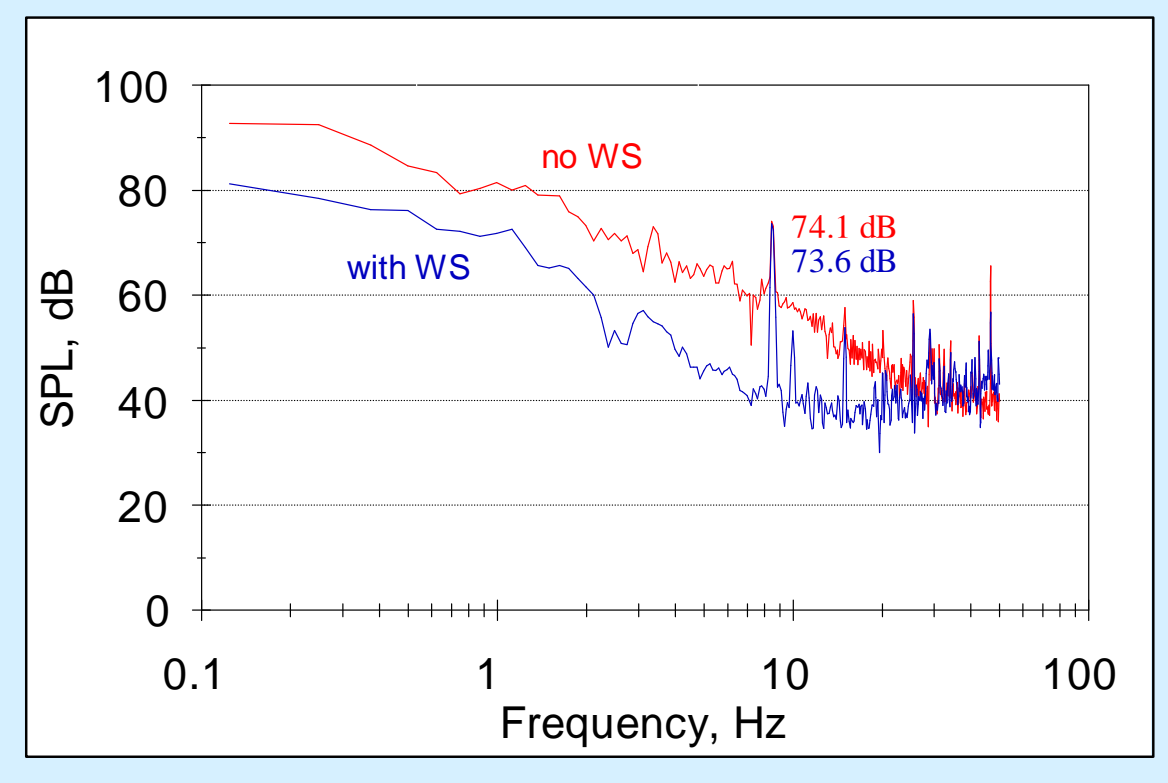

Net gain $-0.5 \mathrm{~dB}$
$15 \mathrm{lb}$ foam

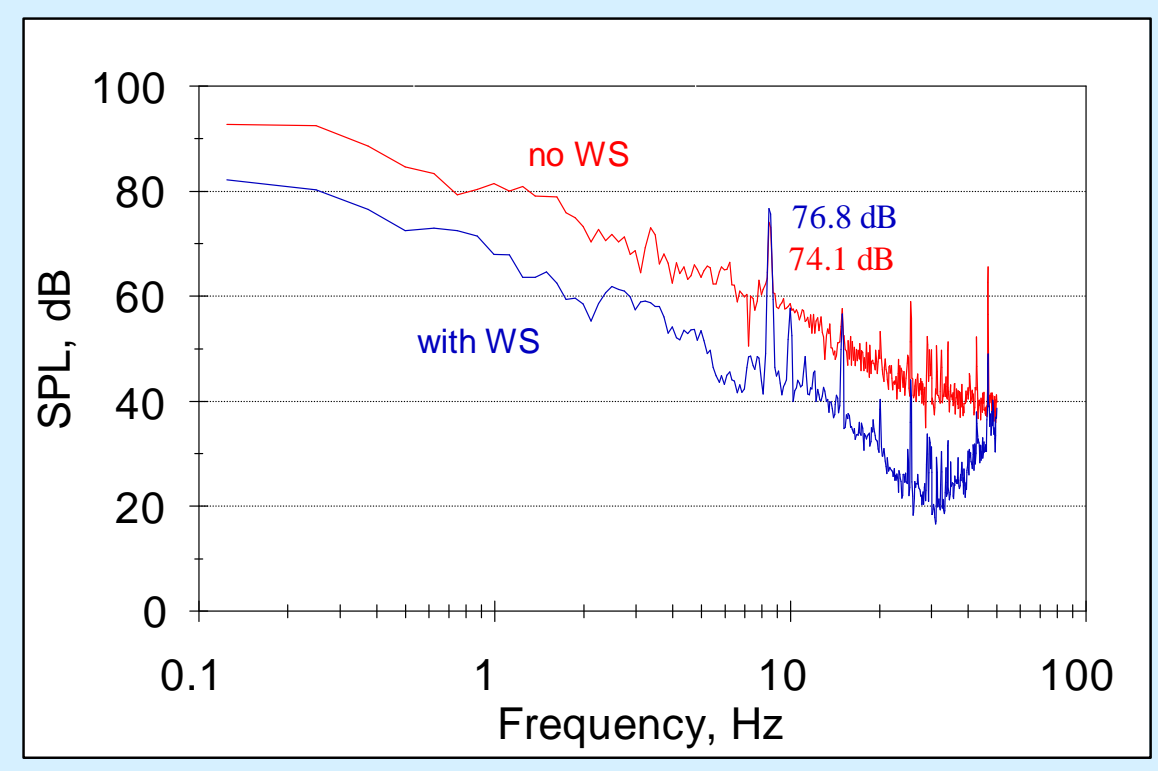

Net gain $2.7 \mathrm{~dB}$ 


\section{Summary: wind noise reduction}

\begin{tabular}{|c|c|c|c|c|}
\hline \multirow{2}{*}{$\begin{array}{c}\text { Wind } \\
\text { speed } \\
\mathrm{m} / \mathrm{s}\end{array}$} & \multicolumn{4}{|c|}{$\begin{array}{c}\text { Wind noise reduction, dB } \\
\text { (mean } 0.1-10 \mathrm{~Hz} \text { ) }\end{array}$} \\
\cline { 2 - 5 } & $\begin{array}{c}4 \mathrm{lb} \\
\text { foam }\end{array}$ & $\begin{array}{c}8 \mathrm{lb} \\
\text { foam }\end{array}$ & $\begin{array}{c}15 \mathrm{lb} \\
\text { foam }\end{array}$ & $\begin{array}{c}4 \times 50-\mathrm{ft} \\
\text { soaker } \\
\text { hose }\end{array}$ \\
\hline $1-2$ & & 16.6 & 20.5 & 10.4 \\
\hline 3 & 15.8 & 16.6 & 17.2 & \\
\hline 5 & 16.4 & 16.1 & 12.6 & \\
\hline 7 & 12.7 & 15.6 & 12.6 \\
\hline
\end{tabular}




\section{Summary: transmission thru windscreen@ $8.55 \mathrm{~Hz}$}

\section{Transmission gain, $\mathrm{dB}$}

$4 \mathrm{lb}$ foam $8 \mathrm{lb}$ foam $15 \mathrm{lb}$ foam $4 \times 50-\mathrm{ft}$ soaker hose

$-0.5$

$+1.0$

$+2.7$

? 